Corresponding author: Atanaska Andreeva

Address: Sofia University, Faculty of Physics,

Department of Condensed Matter Physics,

5, J. Bourchier blvd.,

1164 Sofia, Bulgaria

e.mail: andreeva@phys.uni-sofia.bg

FAX: +3592962576

Phone: +3592 8161847 


\title{
Selective photobleaching of chlorophylls and carotenoids in Photosystem I particles under high-light treatment
}

\author{
Atanaska Andreeva ${ }^{\mathrm{a}^{*}}$, Silvia Abarova ${ }^{\mathrm{a}}$, Katerina Stoitchkova ${ }^{\mathrm{a}}$, Rafael Picorel ${ }^{\mathrm{b}}$ and \\ Maya Velitchkova ${ }^{\mathrm{c}}$
}

\footnotetext{
${ }^{a}$ Sofia University, Faculty of Physics, Department of Condensed Matter Physics, 5, J. Bourchier blvd., 1164 Sofia, Bulgaria.

${ }^{b}$ Aula Dei Experimental Station (CSIC), P.O. Box 202, 50080 Zaragoza, Spain.

${ }^{c}$ Institute of Biophysics, Bulgarian Academy of Sciences, Acad. G. Bonchev str. bl.21, 1113 Sofia, Bulgaria.
} 


\begin{abstract}
Photosystem I particles (PSI-200) isolated from spinach leaves were studied by means of absorbance, $77 \mathrm{~K}$ fluorescence, and resonance Raman spectroscopy. The aim was to obtain better insight into the changes of in the pigment spectral properties of pigments molecules in those Photosystem I particles during prolonged exposure to highlight intensities and to reveal the involvement of these pigments in the photoprotection of the Photosystem I. particles. During prolonged exposure to high-light intensities of spinach Photosystem I particles, a loss of a significant amount of photosynthetic pigments was observed. It was shown that various pigments exhibited different susceptibility to photodamage. effect. In addition to bleaching of chlorophyll $a$, bleaching of carotenoids was also clearly observed. Resonance Raman technique allowed us to recognize the type and conformation of photobleached carotenoid molecules. Raman data revealed a nearly full photobleaching of the long-wavelength lutein molecules. The observed similar bleaching rate of the lutein molecules and the most red-shifted longwavelength chlorophyll $a$ absorbing pigments, located in the antenna membrane protein Lcha4, suggested that these molecules are located closely. Our results showed that the photobleached antenna pigments and especially luteins and the long-wavelength absorbing chlorophylls, being dimers or pigment aggregates, are involved in photoprotection of PSI core complex.
\end{abstract}

Keywords: Carotenoid, chlorophyll, photobleaching, PSI-200, photoprotection, xanthophyll. 


\section{Introduction}

Photosystem I (PSI) of higher plants is a supramolecular pigment-protein complex, located preferentially in the non-appressed regions of thylakoid membranes. It consists of chlorophyll (Chl) $a$ binding core complex (PSI core) where the charge separation takes place and $\mathrm{Chl} a / b$ binding peripheral light-harvesting complex (LHCI) located on one side of the core [1-3]. The PSI core binds approximately 90-100 Chl $a$ and 14-20 $\beta$-carotene molecules [1-3]. The LHCI consists of four different membrane proteins (Lhca1-4), which bind about $110 \mathrm{Chl} a$ and all Chl $b$ molecules and approximately 20 xanthophyll molecules $[3,4]$. The composition and the configuration of the xanthophylls in the LHCII complex have been studied in great details $[5,6]$. In contrast, data about the content and conformational state of carotenoids in the LHCI are scarce and controversial to some extent. Relatively low amounts of neoxanthin and higher levels of lutein, violaxanthin, and $\beta$-carotene are reported for LHCI and PSI core complex from barley, while low amounts of neoxanthin, some $\beta$-carotene (if the $\beta$ carotene is the only pigment beside Chl $a$ in the PSI core why its amount is so low?? Please check it!!) and higher content of lutein and violaxanthin have been obtained in Arabidopsis thaliana [7-9]. Data about carotenoid composition and configuration in spinach PSI particles have been reported recently [10]. The knowledge of carotenoid composition and organization of light-harvesting and core complexes is important for understanding in detail the mechanisms of carotenoid functions in the photosynthetic apparatus. The protection against high-light stress and reactive oxygen species (ROS), realized via the quenching of electronic excited states of Chl $a$ molecules, quenching of 
singlet oxygen and scavenging of free radicals, is one of the main functions of carotenoids [11-13].

Light is required for photosynthesis, but excessive light may cause the inactivation of photosynthesis. Photosynthetic apparatus has evolved multiple photoprotective mechanisms as mentioned above to cope with the potentially damaging effect of light. However, despite these photoprotective defenses, photoinhibition occurs. Although it was believed that PSI is tolerant to strong light, it has recently been shown that PSI can also be photoinhibited [14,15]. Besides the process of photoinhibition, photobleaching of photosynthetic pigments was also observed under high-light conditions. Photobleaching of photosynthetic pigments under high-light illumination is a well-known phenomenon and involves a loss of bulk photosynthetic pigments $[16,17]$. During photoinhibition of isolated PSI complexes, the light-harvesting complex of PSI was more affected than the core complex [17]. A preferential photobleaching of Chl $a$ under high-light treatment was shown for isolated thylakoid membranes [16], PSI and PSII submembrane fractions [18], and PSI reaction center complex [19]. It was shown that Chl $a$ and total carotenoids bleached more intensively in PSI submembrane fraction in comparison with PSII fraction [18]. However, no data about the extent of bleaching of different xanthophylls are available and the mechanism of their participation in high-light protection of plants is still under discussion. On the basis of the time course of photobleaching of chlorophylls during high-light treatment of PSI particles, it was supposed that a slower bleaching at the beginning of illumination could be due to oxidative decomposition of carotenoids [20], but no xanthophylls were specified. Resonance Raman (RR) spectroscopy gives the opportunity to investigate the bleaching 
behavior of different carotenoids in PSI particles and their accessibility to high-light treatment. When the excitation by narrow laser lines coincides with a carotenoid absorption band (in the blue-green region) a $10^{6}$-fold increase in its Raman signal is achieved relative to the scattering due to any other molecules present $[5,21]$, and the spectrum of this carotenoid is observed almost exclusively. This together with the fact that Nevertheless that the position of the most intensive band $v_{1}$ in carotenoid RR spectra is influenced to some extent by the excitation laser lines (as far as I know the frequency of the RR bands are mainly independent of the laser lines; the relative intensity of the RR bands is dependable of the laser lines!!), it was shown that the $v_{1}$ mode position strongly depends on the type and conformation of a specific carotenoid [5], make RR technique suitable provides the possibility to extract the signal from a specific bound carotenoid in complex systems. Recently, based on the selectivity of RR [21] and the established strong dependence of the position of the most intensive band $v_{1}$ in carotenoid RR spectra [5], the absorption transitions for lutein, violaxanthin, and 9-cis neoxanthin in spinach PSI particles were characterized [10]. Therefore, knowing which carotenoid dominates the RR spectra obtained at a given wavelength we can recognize the type and conformation of the photobleached carotenoid molecules.

In the present investigation, PSI particles isolated from spinach leaves were studied by means of electronic absorbance, $77 \mathrm{~K}$ fluorescence and resonance Raman spectroscopy. The aim was to obtain better insight into the changes in the spectral properties of pigment molecules in PSI particles during prolonged exposure to high-light intensities and to reveal the involvement of these pigments in the photoprotection and photoinactivation of PSI particles. 


\section{Experimental details}

\subsection{Isolation of Photosystem I particles}

Photosystem I particles were isolated from spinach chloroplasts by mild digitonin treatment [22]. The submembrane particles obtained by this method originated mainly from stroma exposed regions of thylakoids and consist of PSI core complex and LHCI, so called PSI-200 complexes. The final pellet after the last ultracentrifugation was resuspended in a medium containing $20 \mathrm{mM}$ Tricine- $\mathrm{KOH}(\mathrm{pH} 7.8), 10 \mathrm{mM} \mathrm{NaCl}, 10$ $\mathrm{mM} \mathrm{NaCl}$, and $5 \mathrm{mM} \mathrm{MgCl}_{2}$. Pigment concentration was determined by using the method of Lichtenthaler [23].

\subsection{High-light treatment}

The illumination of isolated PSI particles was carried out in a temperaturecontrolled vessel under continuous stirring at room temperature $\left(22^{\circ} \mathrm{C}\right)$. The $\mathrm{Chl}$ concentration during illumination was $500 \mu \mathrm{g} / \mathrm{ml}$. Samples were placed in flat vessel to ensure equal illumination of all layers of the sample. High intensity illumination was provided by $1000-\mathrm{W}$ halogen projector lamp giving intensity on the vessel surface of $1800 \mu \mathrm{E} \mathrm{m} \mathrm{m}^{-2} \mathrm{~s}^{-1}$. The light was passed through a heat filter. Samples for analysis were taken at different time period $(30,60,90$, and $120 \mathrm{~min})$ during illumination.

\subsection{Electronic absorption measurements and spectral analysis}

Absorption spectra of control and treated PSI particles were performed with a Beckman DU-640 spectrophotometer at room temperature in 1-cm pathlenght cuvette. The Chl concentration during measurements was $15 \mu \mathrm{g} / \mathrm{ml}$. The spectra were analyzed using an Origin 6.0 (Microcal Softwear Inc., USA). 


\subsection{K Chl fluorescence measurements and Gaussian decomposition of}

emission spectra

Samples taken from the temperature-controlled vessel at different time intervals of illumination were transferred into a translucent Dewar and immediately frozen in liquid nitrogen. The $77 \mathrm{~K} \mathrm{Chl} \mathrm{fluorescence} \mathrm{emission} \mathrm{spectra} \mathrm{were} \mathrm{measured} \mathrm{with} \mathrm{a} 0.85 \mathrm{~m}$ double spectrophotometer Spex (model 1403; Spex Industries, Inc., Edison, NJ, USA), equipped with a cooled photomultiplier tube (model R943, Hamamatsu Photonics, K. K., Shizuoka, Japan), as described in [24]. The fluorescence was excited with the by visible line at $488 \mathrm{~nm}$ of an Ar+ laser INNOVA 307 (Coherent). The used power was 1-2 mW and the spectral resolution was $0.15 \mathrm{~nm}$. The experimental spectra were corrected for the spectral sensitivity of the detection system. The Chl content of the samples was adjusted to $10 \mu \mathrm{g} / \mathrm{ml}$.

The steady-state emission properties of PSI-200 complexes at low temperatures (below $80 \mathrm{~K}$ ) are determined by the so called long-wavelength absorbing pigments (LWP) - pigments that absorb at wavelengths longer than the corresponding reaction centers $[2,7,25,26]$. Two LWP pools, emitting mainly at 720 and $735 \mathrm{~nm}$, named F720 and F735, according to their fluorescence maxima are generally found. They are located in PSI core and LHCI-730, respectively $[2,25,26]$. The very low intensity shoulder around 680-690 $\mathrm{nm}$, denoted as F690, is also found and ascribed to LHCI-680 [2,7]. The decomposition of steady-state fluorescence emission spectra of PSI particles was performed by using the curve-fit program included in the packaged Lab calc, Arithmetic A2.23 (Galactic Ind. Corp.) programs with three Gaussian sub-bands, corresponding to the three main emission bands F690, F720, and F735 using the least square method. 
The fitting parameters for the amplitudes of three main Gaussian components were only free-running parameters, while the other parameters, their position and full width at the half maximum (FWHM), were fixed in the limits of the literature data $[2,7,25-27]$. The fixed parameters used for the decomposition of $77 \mathrm{~K}$ fluorescence emission spectra in PSI particles are given in Table I.

Table I. Gaussian parameters of bands ascribed to the main pools after the decomposition of the $77 \mathrm{~K}$ fluorescence emission spectra of PSI particles.

\begin{tabular}{ccccc}
\hline Band & \multicolumn{2}{c}{ Center } & \multicolumn{2}{c}{ FWHM } \\
& $\mathrm{nm}$ & $\mathrm{cm}^{-1}$ & $\mathrm{~nm}$ & $\mathrm{~cm}^{-1}$ \\
\hline F690 & 689 & 14512 & 12 & 246 \\
F720 & 724 & 13812 & 21 & 400 \\
F735 & 738.5 & 13540 & 26 & 480
\end{tabular}

\section{5. Resonance Raman spectra measurements}

For resonance Raman measurements the Chl concentration was $500 \mu \mathrm{g} / \mathrm{ml}$. RR spectra at $77 \mathrm{~K}$ were obtained in a translucent Dewar using the same spectrophotometer Spex as in 2.4. Excitation was provided by an Ar+ laser (Innova 307, Coherent) using the 514.5 and $488 \mathrm{~nm}$ lines. Five to ten successive Raman spectra were averaged for each experiment. To compare absolute RR amplitudes, exactly the same amount of Chl in each sample was used and signal arising from the buffer at the $814 \mathrm{~cm}^{-1}$ was used for normalization of the measured spectra.

\section{Results}

3.1. Changes in the electronic absorption spectra 
The absorption spectra of control and illuminated samples are compared in Fig. 1 (a). The spectrum of untreated PSI particles showed typical peaks at 438 and $680 \mathrm{~nm}$ corresponding to absorption of $\mathrm{Chl} a$, and a shoulder at $650 \mathrm{~nm}$ originating from $\mathrm{Chl} b$. The positions of the main peaks were verified by analysis of the first derivative of the absorption spectra in the blue [Fig.1 (b)] and the red [Fig. 1 (c)] regions. After $120 \mathrm{~min}$ illumination a blue shift of approximately $6 \mathrm{~nm}$ of the Chl $a$ maximum at $678 \mathrm{~nm}$ is observed, whereas the shift of the peak at $438 \mathrm{~nm}$ is only $2 \mathrm{~nm}$. The pronounced shoulder in the blue-green region is related to absorption of $\mathrm{Chl} b$ and carotenoids. In illuminated PSI particles, the intensity of the absorption of Chl $a$ at $438 \mathrm{~nm}$ decreased gradually with the time of illumination showing less than half intensity after $120 \mathrm{~min}$ illumination compared to the untreated samples. In the blue-green region the decreasing is even more pronounced and in fact after 120 min illumination the main shoulder at around $490 \mathrm{~nm}$ almost disappeared. As the absorption at $650 \mathrm{~nm}$ due to $\mathrm{Chl} b$ is less affected, the strong decrease in the blue-green region is a result of the predominant photobleaching of carotenoids. This becomes particularly clear from the changes in the first derivative spectra shown in Fig.1 (b). The long-wavelength carotenoid band is considerably diminished and the resulting maximum is blue shifted.

\subsection{Changes of pigment content}

Pigment content of treated samples was determined during illumination in $80 \%$ aqueous acetone by the method of Lichtenhaler [23]. High-light induced changes in the content of Chl $a$, Chl $b$, and total carotenoids are presented in Fig. 2. Chl $a$ content strongly decreases with the time of illumination, while Chl $b$ content remains almost the 
same. The carotenoid content also decreases considerably, although these data cannot determine precisely which type of carotenoid is more affected by light treatment.

\subsection{Changes in $77 K$ fluorescence spectra}

The steady-state emission spectra of PSI particles at $77 \mathrm{~K}$ upon $488 \mathrm{~nm}$ excitation are compared in Fig. 3 (a). The maximum of untreated PSI particle emission spectrum is located nearly at $734 \mathrm{~nm}$. It is typical for spinach PSI fluorescence at low temperature dominated by the emission from LHCI-730 with emission maximum at 730-735 nm [2]. The high-light treatment results in a remarkable blue-shift of the main fluorescence maximum, indicating that fluorescence from the LWP pool emitting at $735 \mathrm{~nm}$, located in LHCI-730, is considerably quenched. In order to analyze in detail the emitting contribution of the distinct $\mathrm{Chl}$ pools as well as their behavior during illumination, the spectra were decomposed as it was described in 2.4. As an example of such decomposition, a typical fit is shown in Fig. 3 (b). Dependencies of the component areas (F690, F720, and F735) on the time of illumination are presented at Fig. 3 (c). At the beginning of illumination, considerable decrease of F735 is observed indicating intensive bleaching of LWP735. At prolonged light treatment (after $45 \mathrm{~min}$ ), F720 also decreases. Upon illumination for 1 hour, the F735 decreases 15 times, F720 - 2.4 times, while F690 increased approximately twice [Fig. 3 (c)]. It can be concluded, therefore, that during prolonged exposure to high-light intensities F735 from LHCI is quenched first, then the F720 from PSI core also decrease, while the fluorescence emitted at $690 \mathrm{~nm}$ is nearly unaffected.

\subsection{Changes in the resonance Raman spectra}


We used RR scattering in an attempt to recognize the type and conformation of photobleached carotenoid molecules. Resonance Raman spectra were measured with excitation at 488 and $514.5 \mathrm{~nm}$ lines, which coincide with the absorption maxima of 9-cis neoxanthin and long-wavelength lutein, respectively $[5,6,10,21]$. The RR spectra contain four known main groups of bands (called from $v_{1}$ to $v_{4}$ ) characteristic of carotenoids [5,10,21] (Fig. 4) and the band at $814 \mathrm{~cm}^{-1}$ arising from the buffer used (B). The main carotenoid bands have been assigned [5,21] as follows: $v_{1}-$ to $\mathrm{C}=\mathrm{C}$ bonds in phase stretching vibrations; $v_{2}-$ to $\mathrm{C}_{14}-\mathrm{C}_{15}$ stretches coupled to $\mathrm{C}_{15}-\mathrm{H}$ in plane bending; $v_{3}-$ to methyl $\mathrm{CH}_{3}$ in plane rocking vibrations; $v_{4}-$ to $\mathrm{C}-\mathrm{H}$ out of plane bending modes coupled with $\mathrm{C}_{7}=\mathrm{C}_{8}$ torsion. The most intensive band $v_{1}$ in the carotenoid RR spectrum is located at $1526.5 \mathrm{~cm}^{-1}$ and $1533 \mathrm{~cm}^{-1}$ upon excitation lines at 514.5 and $488 \mathrm{~nm}$, respectively. The absolute RR band amplitudes are normalized as explained in section 2.4 and their values as a function of the time of illumination are compared in Fig. 5 and 6 for lutein and 9-cis neoxanthin molecules, respectively, where (a) concerns $v_{3}-v_{4}$ region and (b) $v_{1}$ band.

The dependency of $v_{1}$ band intensity on the time of illumination are compared in Fig. 7. It reveals that during high-light treatment the intensity of long-wavelength absorbing lutein $v_{1}$ band decreases drastically, more than 11 times, while the neoxanthin intensity remains nearly the same.

\section{Discussion}

Prolonged exposure to high-light intensities induced a remarkable loss of photosynthetic pigments of spinach PSI particles. However, various pigments exhibited different susceptibility to photodamage. In addition to Chl $a$ bleaching, carotenoids were 
also highly affected by light treatment. In this respect, the great selectivity of RR technique gave us a great opportunity to distinguish between different xanthophylls photodamage and in this way we were able to throw more light on their photoprotective function in PSI complexes of higher plants.

Absorption spectroscopy revealed that the LWP Chl molecule pools bleached extensively under high-light. The observed blue-shift of approximately $6 \mathrm{~nm}$ of the $\mathrm{Chl} a$ maximum at $678 \mathrm{~nm}$ [Fig. 1(c)] and the shift of only $2 \mathrm{~nm}$ of the peak at $438 \mathrm{~nm}$ [Fig. 1(b)] strongly suggested a preferential bleaching of Chl molecules absorbing at longer wavelengths (but I think that normally the electronic transition bands in the UV-Soret region are more compact that the corresponding bands in the red, so the shifts are expected to be also higher in the red!!). 77K fluorescence spectra (Fig. 3) confirmed that LWP pools were totally bleached. In addition, they revealed that the pool of the most redshifted LWP, emitting mainly at $735 \mathrm{~nm}$ (F735) and located in LHCI-730, was bleached first while the fluorescence of LWP pigments emitting at $720 \mathrm{~nm}$ (F720) and located in PSI core, decreased lately. In contrast, the fluorescence originating from LHCI-680 was nearly unaffected or slightly increased. It was reported that Lhca3 and Lhca4 were enriched with extreme red-absorbing chlorophylls [28] and that the pool of spectrally distinct most red-shifted LWP in LHCI was bound specifically to the Lhca4 [29]. It could be assumed, therefore, that pigments bound to these subunits of LHCI were the most affected by high-light treatment. On the basis of the recent analysis of protein degradation during high-light treatment at $4^{0} \mathrm{C}$, by Rajagopal et al. [30] showed that the sequence of changes in Lhca proteins was as follow: Lhca2>Lhca1>Lhca3>Lhca4, being Lhca2 the most affected. The migration of excitation energy was towards holochromes with lower 
energy and Chl molecules with a red-shifted absorption maximum (located in Lhca3 and Lhca4) bleached first. Thus, long-wavelength absorbing pigments are situated in more stable protein subunits and they could be involved in photoprotection. Having in mind that the LWP are most probably Chl $a$ dimers [2,25], our results also indicate that chlorophyll-chlorophyll interactions are very vulnerable to strong illumination (but I think that $\mathrm{Chl}$ dimeric interactions are the basis of the pigment red-shift, and therefore those LWP receive more excitation energy from all higher energy pigments provoking their preferential bleaching!!). The nearly unaffected fluorescence F690 originating from LHCI-680 (located in heterodimer Lhca2-Lhca3) showed that their emitting pigments, being Chl $a$ monomers, are more stable and supports the above supposition. The F690 slightly increase after 60 min illumination might be due to a reduction of energy transfer from these pigments after bleaching of LWP molecule pools. The Lhca subunits form two protein heterodimers: Lhca1-Lhca4 and Lhca2-Lhca3. Bleaching of LWP pigments in both protein heterodimers could serve not only as a protective tool but also as a regulator of light energy supply to the of PSI core complex.

Analysis of the absorption spectra in the blue region (Fig. 1) showed that disappearance of the shoulder around $490 \mathrm{~nm}$ was mainly due to bleaching of carotenoid molecules. Our results are in line with recent observations of fast carotenoid pool bleaching in PSI particles [30] and in thylakoids [31]. Resonance Raman investigation of carotenoid bleaching (Figs. 4-7) allowed us to determine the type and conformation of photobleached carotenoid molecules during the time of illumination. Analysis of RR data revealed nearly a full photobleaching of the long-wavelength lutein molecules, whereas the bleaching of the neoxantin molecules was negligible (Fig. 7). The kinetics of the 
lutein photobleaching [Fig. 7 (c)] and the pool of the most red-shifted LWP fluorescence quenching [Fig. 3 (c)] was nearly the same. The similar rate of bleaching of the LWP located in Lcha4 and Lcha3??, I understood that F735 comes from both Lcha4 and Lcha3!! and long-wavelength lutein molecules supposes that they are located closely. The attempt to assign various carotenoid molecules to different Lhca subunits is still open as the structural models for PSI presented sometime ago [32] did not discuss the positions of these molecules within the structure. Only recently [33], in the "atomic" computational model of higher plant PSI, based on the X-ray structure of PSI from pea [32] and its similarities with the cyanobacterial structure of PSI [34], the positions of carotenoids were proposed. It was supposed that most of $\beta$-carotene molecules occupied similar positions as in cyanobacteria and were located in the interface between PSI and LHCI proteins. They fit well in the binding pockets thus insuring optimal interactions with their protein environment. In this theoretical model, based on the similar protein folding in LHCI and LHCII and on the crystal structure of spinach LHCII at $2.72 \AA$ resolution [35], the authors assumed that two carotenoid molecules were located in Lhca4 and Lhca1 each. The similar rate of bleaching of lutein and LWP located in Lhca4 and Lcha3??, strongly supports this assumption. The obtained results allow us to correlate luteins with the Lhca4 subunit. Having in mind that the helical secondary protein structure of LHCI and LHCII is quite similar $[32,33,35]$, it is reasonable to assume that the lutein molecules in Lhca4 are located similarly as in LHCII complex [35]. It was shown that the polyene chains of the two luteins in LHCII were fixed in two narrow hydrophobic cavities and provided strong and rigid linkage between helices A and B. Moreover, these luteins are located near to $\mathrm{Chl} a 613$ and $\mathrm{Chl} a$ 614, the pair of $\mathrm{Chl} a$ molecules that is thought to 
function as an excitation energy quencher making a poorly fluorescing chlorophyll associate, either a dimer or aggregate $[35,36]$.

Our results indicate that lutein-chlorophyll associations interactions play an important role in photoprotection of PSI particles and confirmed that the photoprotection is an intrinsic property of light-harvesting systems, maybe their "original" function, as pointed out by Jansson [37], and the antenna function appeared latter in evolution. Lutein molecules are located in the LHCI assembled as a belt on one side of the core [32] and their loss will facilitate the access of strong light to the core complex, followed by the inactivation of PSI. Thus, the photobleached antenna pigments and especially luteins and LWP Chl $a$ pools should be involved in photoprotection of PSI complex.

\section{ACKNOWLEDGEMENTS}

This work was supported by the Program "Support for the research activities at the universities" (project № 11/2005) and by the Foundation for the Scientific Research at Sofia University, Bulgaria (project № 74/2006). 


\section{References}

1. J. H. Golbeck and D. A. Bryant, in: Current Topics in Bioenergetics, ed. C. P. Lee, Photosystem I, vol.16 (Academic Press, New York, 1991) p. 83.

2. B. Gobets, R. van Grondelle, Energy transfer and trapping in photosystem I, Biochim. Biophys. Acta, 1507 (2001) 80-99.

3. R. Bassi and D. Simpson, Chlorophyll-protein complexes of barley photosystem I, Eur. J. Biochem. 163 (1987) 221-230.

4. J. P. Thornber, G. F. Peter, D. T. Morishige, S. Gomez, S. Anadan, B. A. Welty, A. Lee, C. Kerfeld, T. Takeuchi and S. Preiss, Light harvesting in photosystem I and II, Biochem. Soc. Trans. 21 (1993) 15-18.

5. A. V. Ruban, A. A. Pascal, B. Robert, P. Horton, Configuration and dynamics of xanthophylls in light harvesting antennae of higher plants, J. Biol. Chem. 276 (2001) 24862-24870.

6. A. V. Ruban, A. Pascal, P. J. Lee, B. Robert, P. Horton, Molecular configuration of xanthophyll cycle carotenoids in photosystem II antenna complexes, J. Biol. Chem. 277 (2002) 42937-42942.

7. V. H. R. Schmidt, S. Potthast, M. Wiener, V. Begauer, H. Paulsen, S. Storf, Pigment binding of photosystem I light-harvesting proteins, J. Biol. Chem. 277 (2002) 37307-37314.

8. T. Morosinotto, S. Castelletti, J. Breton, R. Bassi, R. Croce, Mutation analysis of Lhcal antenna complex, J. Biol. Chem. 277 (2002) 36253-36261.

9. A. Lee, J. P. Thornber, Analysis of the pigment stoichiometry of pigment-protein complexes from barley (Hordeum vulgare), Plant Physiol. 107 (1995) 565-574. 
10. A. Andreeva, M. Velitchkova, Resonance Raman spectroscopy of carotenoids in Photosystem I particles, Biophys. Chem. 114 (2005) 129-135.

11. Gruszecki, W.I., Strzałka, K., Carotenoids as modulators of membrane physical properties, Biochim. Biophys. Acta, 1740 (2005) 108-115.

12. H. A. Frank and R. J. Cogdell, in: Carotenoids in photosynthesis, ed. A.Young and G. Britton, Photochemistry and functions of carotenoids in photosynthesis, (Chapman \& Hall, London, 1993) p. 252.

13. K. K. Nyogi, Photoprotection revisited: Genetic and molecular approaches, Annu. Rev. Plant Physiol. Plant Mol. Biol. 50 (1999) 333-359.

14. K. Sonoike, Selective hotoinhibition of photosystem I in isolated thylakoid membranes from cucumber and sinach, Plant Cell Physiol. 36 (1995) 825-830.

15. Y. Hui, J. Wei, R. Carpentier, Degradation of the Photosystem I Complex During Photoinhibition, Photochem. Photobiol. 72 (2000) 508-512.

16. M. Y. Veltchkova, R. Picorel, Photobleaching of photosynthetic pigments in spinach thylakoid membranes. Effect of temperature, oxygen and DCMU, Biophys. Chem. 107 (2004) 25-30.

17. S. Rajagopal, N.G. Bukhov and R. Carpentier, Photoinhibitory light-induced changes in the composition of chlorophyll-protein complexes and photochemical activity in photosystem I submembrane fractions, Photochem. Photobiol. 77 (2003) 284-291.

18. N. Miller and R. Carpentier, Energy dissipation and photoprotection mechanisms during chlorophyll photobleaching in thylakoid membranes, Photochem. Photobiol. 54 (1991) 465-472. 
19. M. Purcell and R. Carpentier, Homogeneous photobleaching of chlorophyll holochromes in a photosystem I reaction center complex, Photochem. Photobiol. 59 (1994) 215-218.

20. S. Rajagopal, N. G. Bukhov and R. Carpentier, Changes in the structure of chlorophyll-protein complexes and excitation energy transfer during photoinhibitory treatment of isolated photosystem I submembrane particles, Photochem. Photobiol. 62 (2002) 194-200.

21. B. Robert, P. Horton, A. Pascal and A. Ruban, Insights into the molecular dynamics of plant light-harvesting proteins in vivo, Trends Plant Sci. 9 (2004) 385-390.

22. F.A.L.J. Peters, J.E. van Wielink, H.W. Wong Fong Sang, S. De Vries, R.. Kraayenhof, Studies on well coupled photosystem I-enriched subchloroplast vesicles. Content and redox properties of electron transfer components, Biochim. Biophys. Acta 722 (1983) 460-470.

23. H. K. Lichtenthaler, Chlorophylls and carotenoids: Pigments of photosynthetic membranes, Methods Enzymol. 148 (1987) 350-382.

24. A. Andreeva, K. Stoitchkova, M. Busheva, E. Apostolova, The Energy Distribution Between Chlorophyll-Protein Complexes of Thylakoid Membranes from Pea Mutants with Modified Pigment Content I. Changes Due to the Modified Pigment Content, J. Photochem. Photobiol., 70 (2003) 153-162.

25. J. A. Ihalainen, B. Gobets, K. Sznee, M. Brazzoli, R. Croce, R. Bassi, R. van Grondelle, J. E. I. Korppi-Tommola and J. P. Dekker, Evidence for two spectroscopically different dimers of light-harvesting complex I from green plants, Biochem. 39 (2000) 8625-8631. 
26. J. van der Lee, D. Bald, S. L. S. Kwa, R. van Grondelle, M. Rogner and J. P. Dekker, Steady-state polarized light spectroscopy of isolated photosystem I complexes, Photosynth. Res. 35 (1993) 311-321.

27. A. Andreeva, M Velitchkova, Polarized fluorescence spectroscopy of oriented isolated spinach Photosystem I particles, Photosynth. Res. 65 (2000) 15-28.

28. T. Morossinoto, J. Breton, R. Bassi and R. Croce, The Nature of a Chlorophyll Ligand in Lhca Proteins Determines the Far Red Fluorescence Emission Typical of Photosystem I, J. Biol. Chem. 278 (2003) 49223-49229.

29. A. Melkozernov, Excitation energy transfer in Photosystem I from oxygenic organisms, Photosyntesisi Res. 70 (2001) 129-153.

30. S. Rajagopal, D. Joly, A. Gauthier, M. Beauregard and R. Carpentier, FEBS Journal, 272 (2005) 892-902.

31. S. Santabarbara, Limited sensitivity of pigment photo-oxidation in isolated thylakoids to singlet state quenching in photosystem II antenna, Arch. Biochem. Biophys. 455 (2006) 77-88.

32. A. Ben-Shem, F. Frolov and N. Nelson, Crystal structure of plant photosystem I, Nature 496 (2003) 630-635.

33. C. Jolley, A. Ben-Shem, Structure of plant photosystem I revealed by theoretical modeling, J. Biol. Chem. 280 (2005) 33627-33636.

34. P. Jordan, P. Fromme. H. T. Witt, O. Klukas, W. Saenger and N. Krauss, Threedimensional structure of cyanobacterial Photosystem I at $2.5 \AA$ resolution, Nature, 411 (2001) 909-917. 
35. Z. Liu, H. Yan, K. Wang, T. Kuang, J. Zhang, X. An and W. Chang, Crystal structure of spinach major light-harvesting complex at $2.72 \AA$ resolution, Nature 428 (2004) 287-292.

36. A. Pascal, Z. Liu, K. Broess, B. van Oort, H. C. Wang, P. Horton, B. Robert, W. Chang and A. Ruban, Molecular basis of photoprotection and control of photosynthetic light-harvesting, Nature 436 (2005) 134-137.

37. S. Jansson, A protein family saga: from photoprotection to light-harvesting (and back?), in: Photoprotection, photoinhibition, Gene Regulation and Environment, ed. B.Demmig-Adams et al., Dordrecht, The Netherlands: Springer, vol. 21 (2006) $145-153$. 
Legends to figures:

Figure 1. Absorption spectra of spinach PSI particles (a) and their first derivatives in the blue (b) and red (c) spectral regions. Control (line 1) and illuminated for $30 \mathrm{~min}$ (line 2), $60 \mathrm{~min}$ (line 3), and $120 \mathrm{~min}$ (line 4) with a strong white light $\left(1800 \mu \mathrm{E} \mathrm{m}^{-2} \mathrm{~s}^{-1}\right)$.

Figure 2. Pigment content changes of spinach PSI particles during strong illumination (see Materials and Methods). The conditions were the same as in Fig. 1.

Figure 3. 77K fluorescence emission spectra of spinach PSI particles after excitation with the $488 \mathrm{~nm}$ line of an Ar+ laser. (a) Control (line 1) and illuminated for 30 min (line 2), $60 \mathrm{~min}$ (line 3), and $120 \mathrm{~min}$ (line 4) with a strong white light $\left(1800 \mu \mathrm{E} \mathrm{m}^{-2} \mathrm{~s}^{-1}\right)$; (b) A typical fit for the decomposition of the $77 \mathrm{~K}$ emission spectrum into three main bands; (c) Variation of the three component areas as a function of illumination time.

Figure 4. $77 \mathrm{~K}$ resonance Raman spectra in the high frequency region of untreated (line 1) and illuminated for 60 min (line 2) PSI particles upon excitation with the $488 \mathrm{~nm}$ line of an Ar+ laser. Excitation at $514.5 \mathrm{~nm}$ of untreated (line 3) PSI particles.

Figure 5. 77K resonance Raman spectra from spinach PSI particles in the $v_{3}-v_{4}$ (a) and $v_{1}$ (b) regions upon $514.5 \mathrm{~nm}$ excitation of control (line 1) and illuminated for $30 \mathrm{~min}$ (line 2), 60 min (line 3) with a strong white light $\left(1800 \mu \mathrm{E} \mathrm{m}^{-2} \mathrm{~s}^{-1}\right)$. 
Figure 6. $77 \mathrm{~K}$ resonance Raman spectra from spinach PSI particles in the $v_{3}-v_{4}$ (a) and $v_{1}$ (b) regions upon $488 \mathrm{~nm}$ excitation of control (line 1) and illuminated for $30 \mathrm{~min}$ (line 2), $60 \mathrm{~min}$ (line 3), and $120 \mathrm{~min}$ (line 4) with a strong white light $\left(1800 \mu \mathrm{E} \mathrm{m}^{-2} \mathrm{~s}^{-1}\right)$.

Figure 7. Dependence of $v_{1}$ band intensity on the time of illumination of lutein (circles) and neoxanthin (squares) molecules of spinach PSI particles. Data were extracted from the results of Fig. 5 and Fig. 6. 


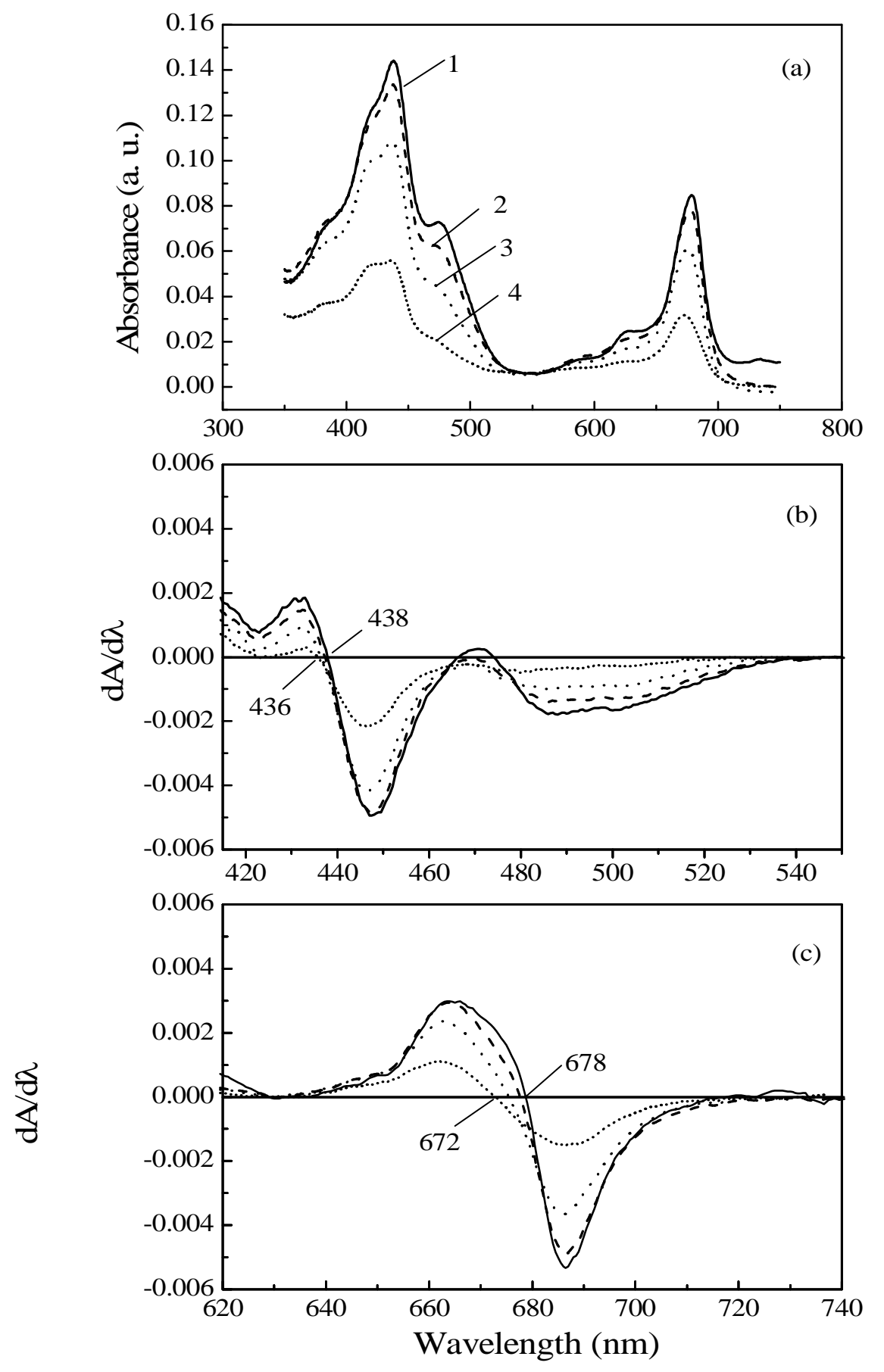

Fig.1 


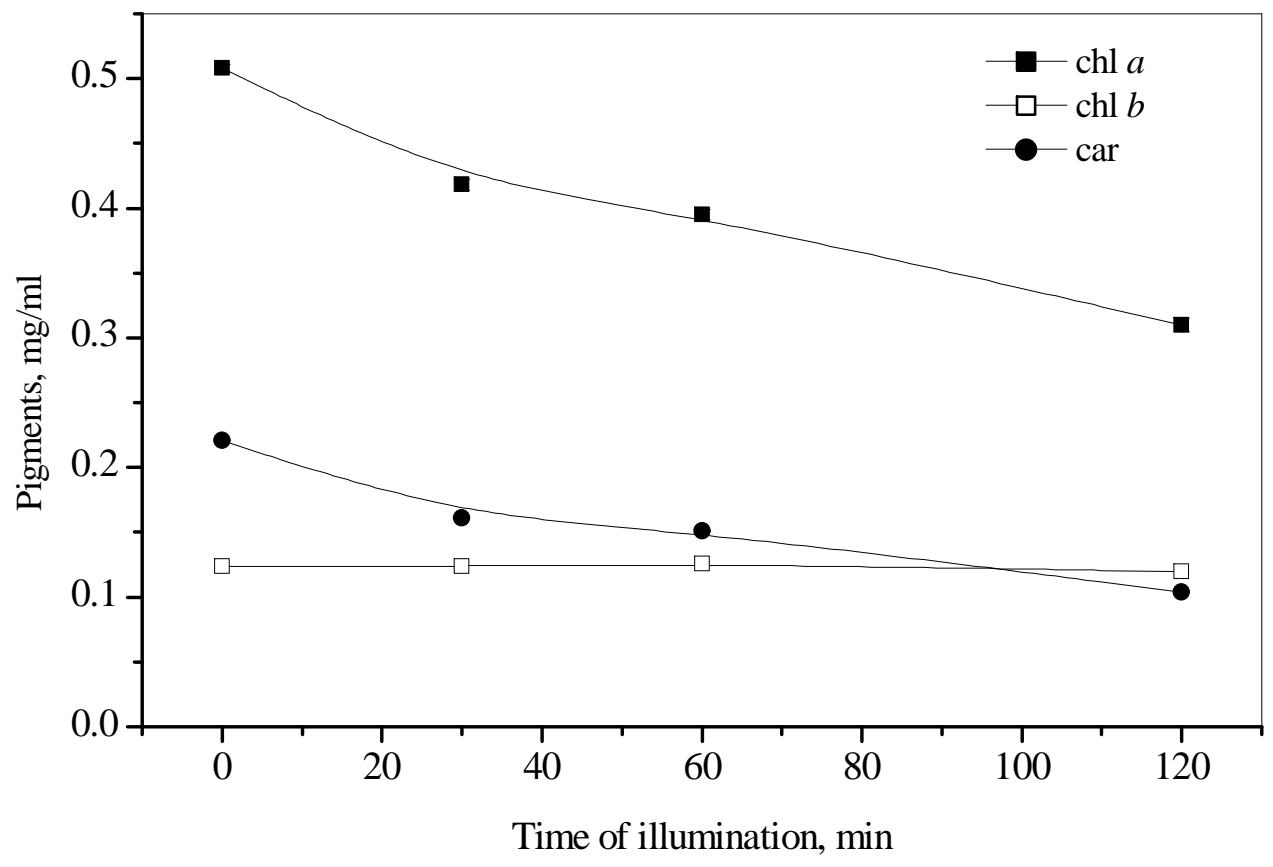

Fig.2 
Emission Wavelength, nm
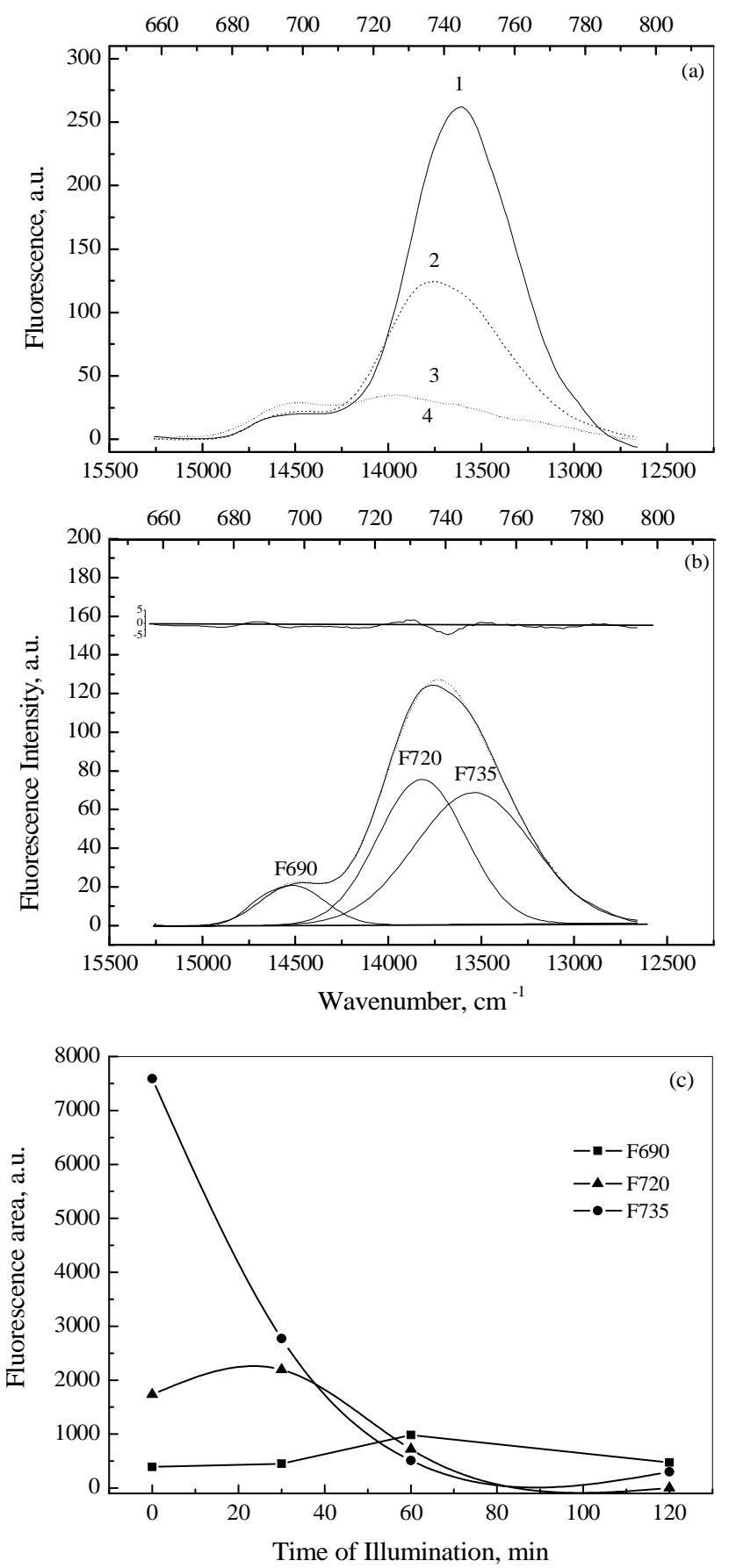

Fig. 3. 


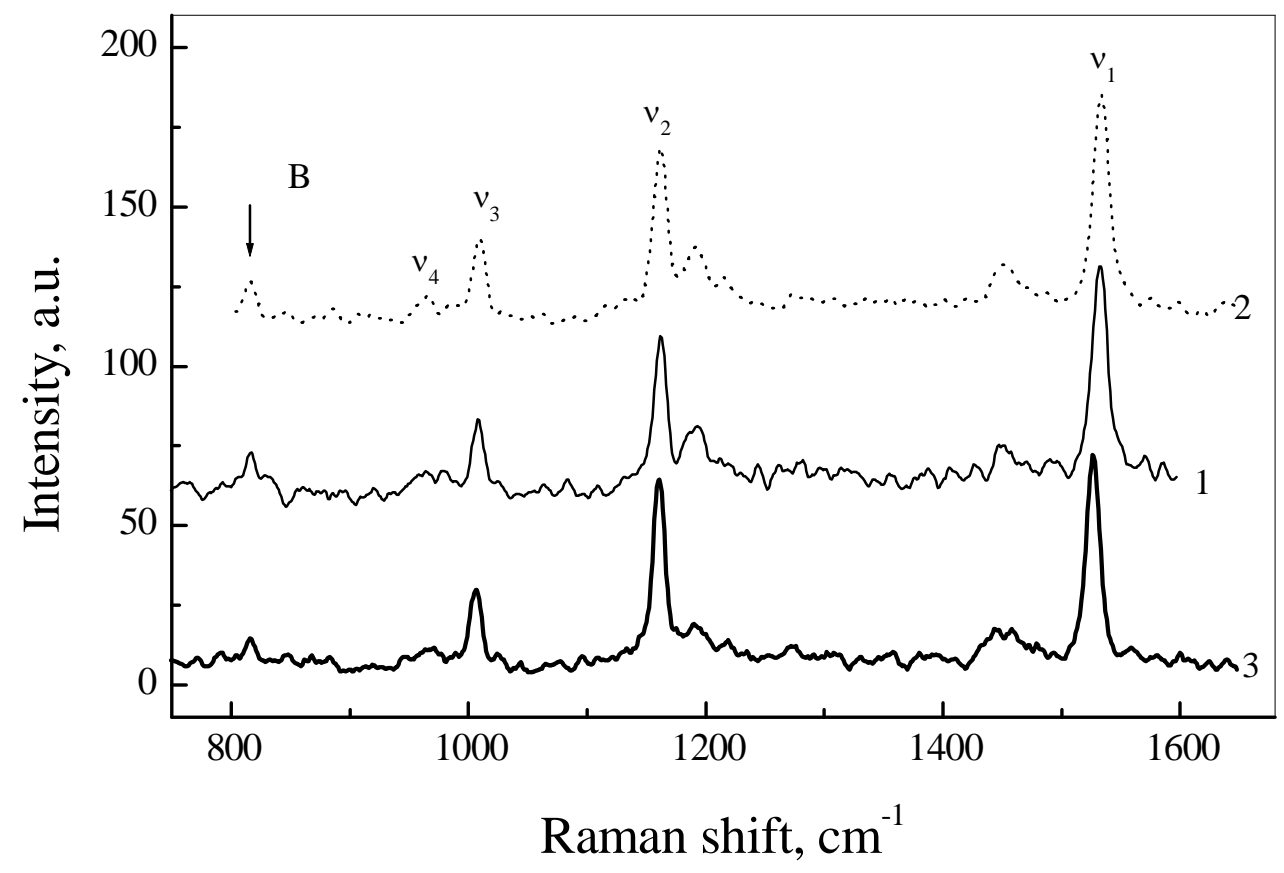

Fig. 4 

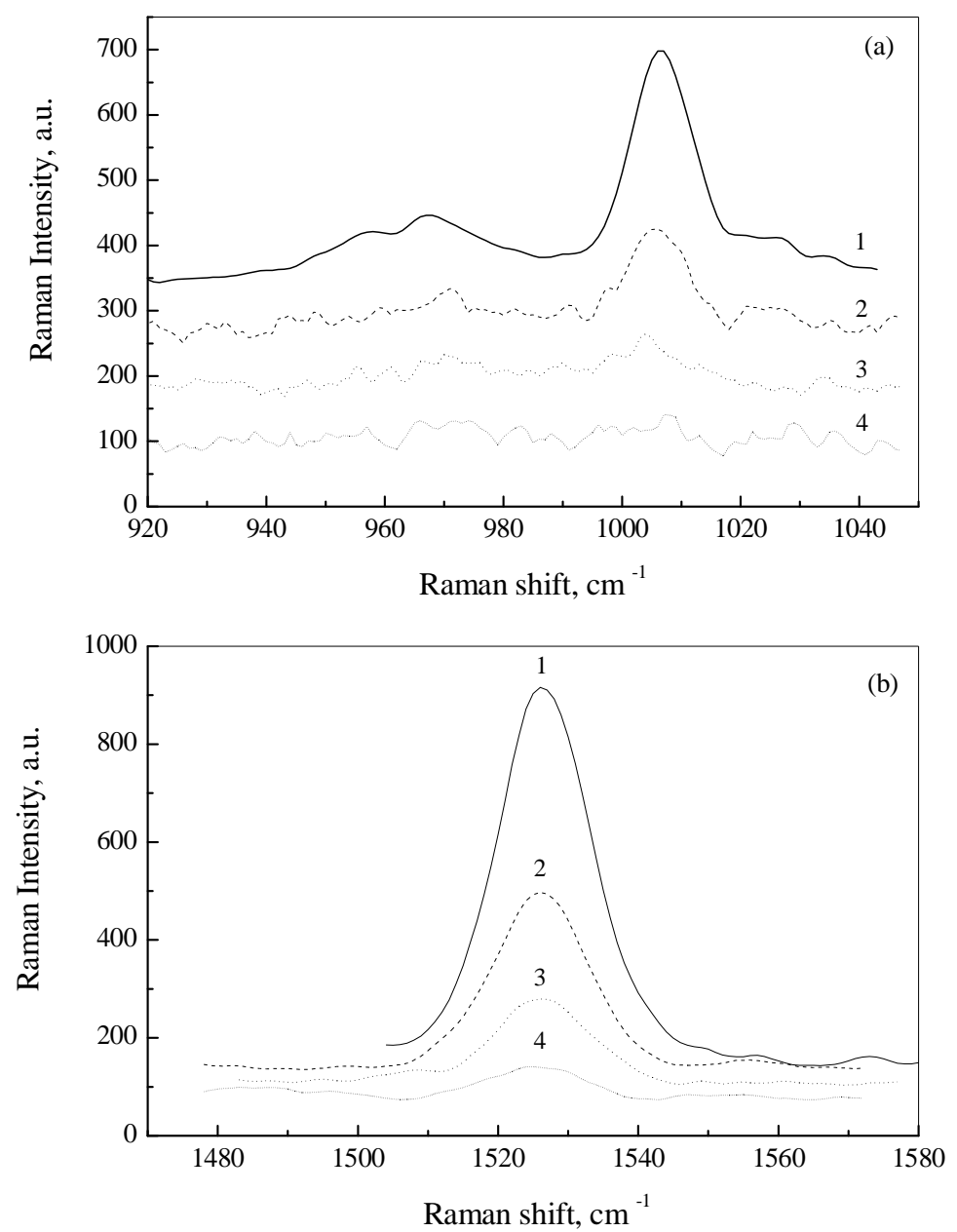

Fig. 5. 

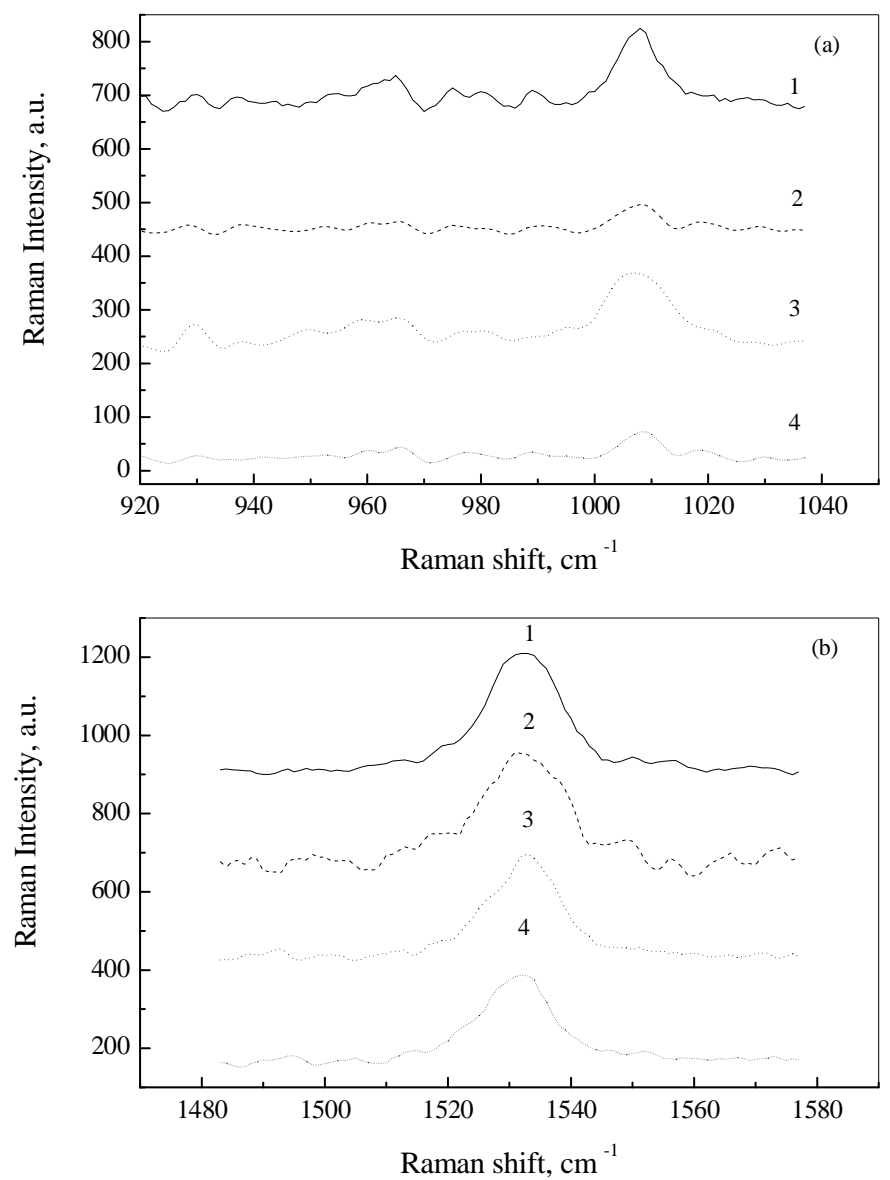

Fig.6. 


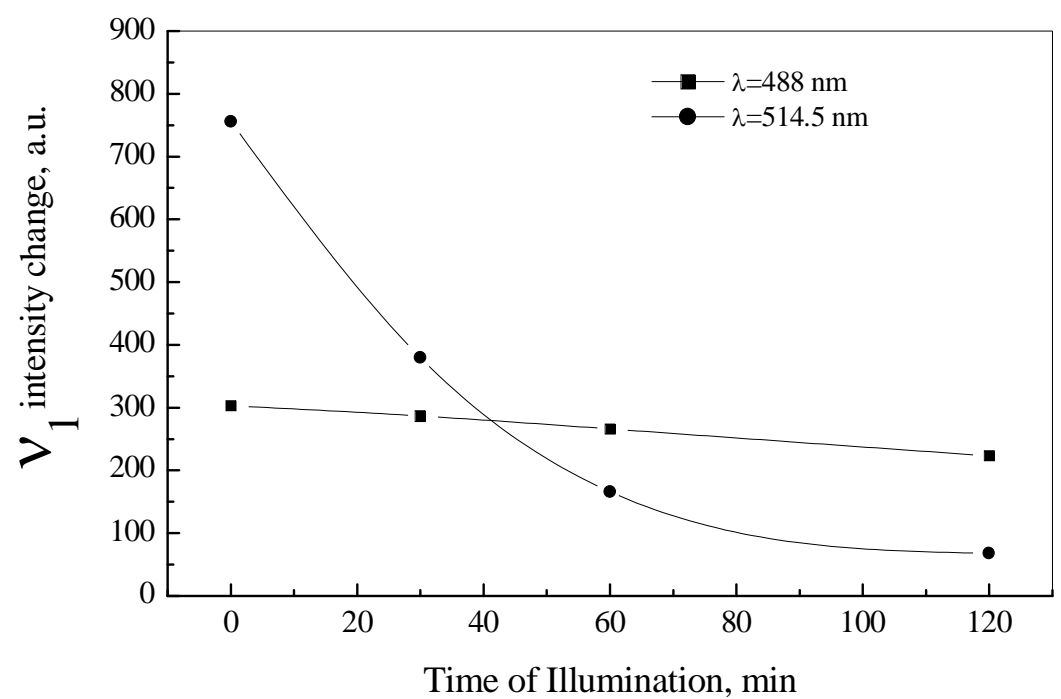

Fig. 7. 